For an even $\lambda$, this becomes

$$
\alpha-\alpha_{2}+\alpha_{4}-\cdots=1 \text {. }
$$

For any $\lambda$, there is $\alpha_{1}-\alpha_{3}+\alpha_{5}-\cdots=0$. When $\lambda$ is odd, then $-\alpha+\alpha_{2}-\alpha_{4}+\cdots=1$. When $n$ is odd, say $n=2 \lambda$ +1 , then $\alpha-\alpha_{2}+\alpha_{4}-\cdots=0$, and $\alpha_{1}-\alpha_{3}+\alpha_{5}-\cdots$ $=1$, when $\lambda$ is odd; $-\alpha_{1}+\alpha_{3}-\alpha_{5}+\cdots=1$, when $\lambda$ is even.

We shall, in particular, consider the case where (15) has the form

$$
w^{n}-v^{2 k} \cdot u^{n-2 k}=0,
$$

in which $n$ and $k$ must both be either even or odd in order that (16) may reduce to (14) for $u=-1, v=-\ddot{i}$.

After a rather complicated process of elimination the cartesian equation of this special class of curves with the $n$th roots of unity as foci becomes

$$
x^{n-2 k} \cdot y^{2 k}=(-1)^{n-2 k} \cdot \frac{(2 k)^{2 k}}{n^{n} \cdot(n-2 k)^{2 k-n}},
$$

which is an $n$-ic. For $n=3, k=1$, we get the cubic hyperbola $x y^{2}=-4 / 27$. The condition for a proper $n$-ic in (17) is evidently $n-2 k \geqq 1, n \geqq 3$.

UNIVERSITY OF ILLINOIS.

\title{
QUADRATIC SYSTEMS OF CIRCLES IN NON-EUCLIDEAN GEOMETRY.
}

\author{
BY PROFESSOR D. M. Y. SOMMERVILLE.
}

(Read before the American Mathematical Society, October 26, 1918.)

$\S 1$. The equation of any circle can be written

$$
k S-\alpha^{2}=0,
$$

where $S=0$ is the equation of the absolute, and

$$
\alpha \equiv l x+m y+n z=0
$$

is the equation of the axis, the center being the absolute polar of this line. 
If the circle belongs to a one-parameter system, the coeffcients $k, l, m, n$ will be functions of the single parameter $\lambda$. The degree of the system is the number of circles which pass through a given point, and its class is the number of circles which touch a given straight line.

If

$$
\begin{aligned}
& S \equiv a_{0} x^{2}+\cdots+2 f_{0} y z+\cdots \equiv(S \zeta x)^{2}=0, \\
& \Sigma \equiv A_{0} \xi^{2}+\cdots+2 F_{0} \eta \zeta+\cdots \equiv(\Sigma \not \xi)^{2}=0
\end{aligned}
$$

represent the point and line equations of the absolute, the line equation of the circle (1) is

$$
\begin{aligned}
& k \Sigma-\left(b_{0} n^{2}+c_{0} m^{2}-2 f_{0} m n\right) \xi^{2}-\cdots \\
& \quad+2\left(f_{0} l^{2}+a_{0} m n-h_{0} n l-g_{0} l m\right) \eta \zeta+\cdots=0
\end{aligned}
$$

which may also be written

$$
\left\{(\Sigma l l)^{2}-k \Delta_{0}\right\} \Sigma-\{\Sigma(A l+H m+G n) \xi\}^{2}=0 .
$$

The system of circles will be of the first degree only if $l, m, n$ are constant, and $k=(a \lambda+b) /(c \lambda+d)$; it is then also of the first class. Hence the only linear system of circles is a concentric system.

$\S 2$. If $l, m, n$ are of degree $r$ in $\lambda$, while $k$ is of degree $2 r$ or $2 r+1$, the system is of degree $2 r$ or $2 r+1$ respectively, and its class is also $2 r$ or $2 r+1$. Hence the degree and class are in general the same.

$\S 3$. We shall confine our attention now to a quadratic system. The most general form may be written

$$
\left(p \lambda^{2}+2 q \lambda+r\right) S-(\lambda \alpha+\beta)^{2}=0,
$$

where $\alpha$ and $\beta$ represent distinct fixed lines, and $p, q, r$ are given constants. If the lines $\alpha$ and $\beta$ coincide, the equation of the system reduces to

$$
\left(p \lambda^{2}+2 q \lambda+r\right) S-(\lambda+k)^{2} \alpha^{2}=0 .
$$

For all values of $\lambda$ this represents a system of circles with axis $\alpha$, i. e., a concentric system. Though apparently a quadratic system, it is really linear, for two values of $\lambda$ correspond to each circle. 
The lines $\alpha$ and $\beta$ may be replaced by any two distinct lines through their point of intersection without in any way altering the system; they might, for example, be the tangents to $S$, or a pair of conjugate lines with regard to $S$.

The axes of the circles form a pencil of lines $\lambda \alpha+\beta=0$ through the fixed point $A \equiv(\alpha \beta)$, and the centers are collinear on the line $a$, the absolute polar of $A$. form

\$ 4. Envelope of the system. Writing the equation (3) in the

$$
\lambda^{2}\left(p S-\alpha^{2}\right)+2 \lambda(q S-\alpha \beta)+\left(r S-\beta^{2}\right)=0,
$$

the equation of the envelope is

i. e.,

$$
(q S-\alpha \beta)^{2}-\left(p S-\alpha^{2}\right)\left(r S-\beta^{2}\right)=0,
$$

$$
\left(q^{2}-p r\right) S^{2}+\left(r \alpha^{2}+p \beta^{2}-2 q \alpha \beta\right) S=0 .
$$

Hence the envelope consists of the absolute $S$ and a conic

$$
K \equiv\left(p r-q^{2}\right) S-\left(r \alpha^{2}-2 q \alpha \beta+p \beta^{2}\right)=0 .
$$

The equation (3) may be written in the form

$$
\left(p \lambda^{2}+2 q \lambda+r\right) K+\{(q \lambda+r) \alpha-(p \lambda+q) \beta\}^{2}=0,
$$

which shows that every circle of the system has double contact with the conic $K$, and the chords of contact all pass through $A$.

§5. Limiting lines. In general there are two circles of the system which degenerate to coincident lines, limiting lines. The values of $\lambda$ are the roots of the equation $p \lambda^{2}+2 q \lambda+r=0$, and the two lines are $r \alpha^{2}-2 q \alpha \beta+p \beta^{2}=0$. These form a pair of common chords of $S$ and the conic envelope $K$, i. e., a pair of focal lines of $K$. Their point of intersection $A \equiv(\alpha \beta)$ is therefore a center of $K$, and its absolute polar $a$ is an axis of $K$. Hence the line of centers of the circles is one of the axes of the conic envelope, and the point of concurrence of the axes of the circles is the corresponding center of the conic envelope.

The two limiting lines are a pair of focal lines of $K$; the centers of these two degenerate circles are a pair of director points of $K$.

$\S 6$. The pairs of corresponding chords of contact of the circles with $S$ and $K$ form an involution whose double lines are the limiting lines.

To the line $\lambda \alpha+\beta=0$ corresponds the line

$$
(q \lambda+r) \alpha-(p \lambda+q) \beta=0
$$


or, say

so that

$$
\mu \alpha+\beta=0,
$$

i. e.,

$$
\mu(p \lambda+q)+(q \lambda+r)=0,
$$

$$
p \lambda \mu+q(\lambda+\mu)+r=0,
$$

a symmetrical lineo-linear relation. The double elements are then given by

$$
p \lambda^{2}+2 q \lambda+r=0,
$$

the roots of which correspond to the two limiting lines.

If the roots of the equation (6) are $c, d$, and the limiting lines are $\gamma, \delta$, the equation of the system of circles can be written

$$
(\lambda-c)(\lambda-d) S-\{\gamma(\lambda-d)-\delta(\lambda-c)\}^{2}=0 .
$$

Then we find that

$$
K \equiv S+4 \gamma \delta
$$

and the equation of the system can be written also

$$
(\lambda-c)(\lambda-d) K-\{\gamma(\lambda-d)+\delta(\lambda-c)\}^{2}=0
$$

which verifies that the two chords of contact of any circle of the system with $S$ and $K$ are harmonic conjugates with regard to $\gamma, \delta$.

From this symmetrical relationship it follows that if one circle has as chords of contact with $S$ and $K$ the lines $u$ and $v$ respectively, there is another circle of the system which has as chords of contact $v$ and $u$ respectively.

$\S 7$. Limiting points. $S$ and $K$ have four common tangents. Any two of these taken together form a degenerate circle having double contact with $S$ and $K$. But of these pairs just one pair have their centers on the given line of centers of the system of circles. Hence there are two circles of the system which degenerate to a line pair (point circles) and their centers form a pair of foci of the conic envelope $K$. We shall call these two points the limiting points of the system.

§ 8. Associated systems. Let $A, B, C$ be the three centers, $a, b, c$ the corresponding axes of the conic $K$, and let $f, f^{\prime}$; $g, g^{\prime} ; h, h^{\prime}$ be the focal lines through $A, B, C$ respectively; $F, F^{\prime} ; G, G^{\prime} ; H, H^{\prime}$ the foci on $a, b, c$ respectively. Then 
there are three systems of circles, one with limiting points $F, F^{\prime}$, and limiting lines $f, f^{\prime}$, the second with limiting points $G, G^{\prime}$, etc.

$\$ 9$. Horocycles. In general the system contains two horocycles, corresponding to the two values of $\lambda$ which make the line $\lambda \alpha+\beta=0$ a tangent to $S$. These values of $\lambda$ are the roots of the quadratic

$$
\lambda^{2}\left(\Sigma l_{1} l_{1}^{2}+2 \lambda\left(\Sigma l_{l} l_{1} l_{2}\right)+\left(\Sigma l_{2}\right)^{2}=0 .\right.
$$

$\S 10$. Degenerate systems. The discriminant of the conic envelope

where

$$
K \equiv\left(p r-q^{2}\right) S-\left(r \alpha^{2}-2 q \alpha \beta+p \beta^{2}\right)=0,
$$

and

$$
S \equiv\left(a_{0} b_{0} c_{0} f_{0} g_{0} h_{0} \backslash x y z\right)^{2},
$$

is

$$
\alpha \equiv l_{1} x+m_{1} y+n_{1} z, \quad \beta \equiv l_{2} x+m_{2} y+n_{2} z,
$$

$$
\begin{aligned}
&\left(p r-q^{2}\right)^{2}\left[\Delta_{0}\left(p r-q^{2}\right)-p\left(\Sigma \chi l_{2}\right)^{2}+2 q\left(\Sigma \chi l_{1}\right)\left(l_{2}\right)\right. \\
&\left.-r\left(\Sigma \not l_{1}\right)^{2}+\left(S \backslash m_{1} n_{2}-m_{2} n_{1}\right)^{2}\right]=0,
\end{aligned}
$$

$\Delta_{0}$ being the discriminant of $S$.

If $p r-q^{2}=0$, the equation of the conic envelope reduces to

$$
r \alpha^{2}-2 q \alpha \beta+p \beta^{2}=0,
$$

which now represents two coincident lines. As an envelope, the conic degenerates to two points. Put $p=k^{2}, q=k$, $r=1$; then the equation of the system of circles (3) becomes

i. e.,

$$
(k \lambda+1)^{2} S-(\lambda \alpha+\beta)^{2}=0,
$$

$$
\lambda^{2}\left(k^{2} S-\alpha^{2}\right)+2 \lambda(k S-\alpha \beta)+\left(S-\beta^{2}\right)=0 .
$$

Now

and

$$
k^{2} S-\alpha^{2} \equiv k^{2}\left(S-\beta^{2}\right)+\left(k^{2} \beta^{2}-\alpha^{2}\right),
$$

$$
k S-\alpha \beta \equiv k\left(S-\beta^{2}\right)+\beta(k \beta-\alpha) .
$$

Hence the three conics $k^{2} S-\alpha^{2}=0, k S-\alpha \beta=0, S-\beta^{2}=0$ have two points in common, their common chord being $\alpha-k \beta=0$. All the circles of the system therefore pass through these two fixed points, and this point pair is the 
degenerate conic envelope $K$. We have then a coaxal system of circles.

When the other factor of the discriminant (7) vanishes, the conic envelope reduces to two distinct lines, and we have a homocentric system of circles with two common tangents. Multiplying the factor by $\Delta_{0}$, the condition can be expressed in the form

$$
\left[\Delta_{0} p-\left(\Sigma \not l_{1}\right)^{2}\right]\left[\Delta_{0} r-\left(\Sigma l l_{2}\right)^{2}\right]-\left[\Delta_{0} q-\left(\Sigma \not l \gamma l_{1} l l_{2}\right)\right]^{2}=0 .
$$

$\S 11$. The system will be specialized also if the conic envelope is a circle. If $K$ is a circle the common chords $r \alpha^{2}-2 q \alpha \beta+p \beta^{2}=0$ must either coincide or be tangents to the absolute. In the former case, however, $K$ degenerates to these two coincident straight lines, or, as an envelope, to a point pair.

The equation of the tangents from the point $(\alpha \beta)$ to the absolute is

$$
\alpha^{2}\left(\Sigma \not l_{2}\right)^{2}-2 \alpha \beta\left(\Sigma \not l_{1} l_{2} l_{2}\right)+\beta^{2}\left(\Sigma \not l_{1}\right)^{2}=0 .
$$

Hence the conditions that $K$ should be a circle are

$$
p=k\left(\Sigma\left(l_{1}\right)^{2}, \quad q=k\left(\Sigma \chi l_{1} \gamma l_{2}\right), \quad r=k\left(\Sigma\left(l_{l} l_{2}\right)^{2} .\right.\right.
$$

Then

$$
p r-q^{2}=k^{2} \Delta_{0}\left(S \zeta m_{1} n_{2}-m_{2} n_{1}\right)^{2},
$$

and the discriminant of $K,(7)$, becomes

$$
k^{4} \Delta_{0}^{2}\left[\left(S \zeta m_{1} n_{2}-m_{2} n_{1}\right)^{2}\right]^{3}\left(k \Delta_{0}-1\right)^{2} .
$$

In this case therefore $K$ does not in general degenerate further unless the point $(\alpha \beta)$ lies on the absolute. Hence the conic envelope may be a circle, but cannot be a horocycle.

When $K$ is a circle its axis is the line of centers of the circles of the system. The common chords with $S$ and $K$, being harmonic conjugates with regard to the two common tangents of $S$ and $K$, are at right angles. The chords of contact with $K$ all pass through $(\alpha \beta)$ and are therefore perpendicular to the axis of $K$. Hence all the circles of the system have equal radii. The system therefore consists of a system of equal circles with collinear centers. The limiting points reduce to the two absolute points of $K$, and the limiting lines to the absolute lines of $K$. 
\$12. Taking two conjugate lines through $(\alpha \beta)$ and the absolute polar of $(\alpha \beta)$ as the triangle of reference, the equation of the general quadratic system of circles can be written

$$
\left(p \lambda^{2}+2 q \lambda+r\right)\left(x^{2}+y^{2}+z^{2}\right)-(\lambda x+y)^{2}=0 .
$$

Let the radius be $\rho$, then

(8) $c^{2}=\cos ^{2} \rho=\frac{(\lambda x+y)^{2}}{\left(x^{2}+y^{2}+z^{2}\right)\left(\lambda^{2}+1\right)}=\frac{p \lambda^{2}+2 q \lambda+r}{\lambda^{2}+1}$.

Hence there are in general two circles of the system with a given radius.

Let the parameters of the two circles be $\lambda_{1}$ and $\lambda_{2}$, which are the roots of the equation

$$
\left(p-c^{2}\right) \lambda^{2}+2 q \lambda+\left(r-c^{2}\right)=0 .
$$

Then

$$
\begin{aligned}
\lambda_{1}+\lambda_{2} & =-2 q /\left(p-c^{2}\right), \\
\lambda_{1} \lambda_{2} & =\left(r-c^{2}\right) /\left(p-c^{2}\right) .
\end{aligned}
$$

Eliminating $c^{2}$, we get

$$
q \lambda_{1} \lambda_{2}-\frac{1}{2}(p-r)\left(\lambda_{1}+\lambda_{2}\right)-q=0 .
$$

Hence the parameters of pairs of equal circles are connected by an involutorial homographic relation.

There are two coincident pairs of equal circles, whose parameters are the roots of the equation

$$
q \lambda^{2}-(p-r) \lambda-q=0,
$$

and it is easily verified that these are the circles of maximum or minimum radius. The radii of these two circles are found by expressing the condition for equal roots in the equation for $\lambda,(9)$; we get then the equation

$$
c^{4}-(p+r) c^{2}+\left(p r-q^{2}\right)=0 .
$$

if these two values of the radius are equal, all the radii will be equal. The conditions for this are $q=0$ and $p=r$. Equation (8) cannot then be satisfied except for a particular value of $c$. 
If there are three circles of the system with equal radii, all the circles of the system must have equal radii.

$\S 13$. Two-parameter system of circles. There is no linear two-parameter system of circles. The general quadratic system can be written in terms of the homogeneous parameters $\lambda, \mu, \nu$

$$
\begin{aligned}
\left(a \lambda^{2}+b \mu^{2}+c \nu^{2}+2 f \mu \nu+2 g \nu \lambda\right. & +2 h \lambda \mu) S \\
& -(\lambda \alpha+\mu \beta+\nu \gamma)^{2}=0,
\end{aligned}
$$

where $\alpha, \beta, \gamma$ represent three straight lines.

If the lines $\alpha, \beta, \gamma$ are concurrent, so that $\gamma \equiv l \alpha+m \beta$, then, putting $\lambda+l \nu=\lambda^{\prime}, \mu+m \nu=\mu^{\prime}$, the equation can be reduced to the form

$$
\left(a^{\prime} \lambda^{\prime 2}+\cdots+2 f^{\prime} \mu^{\prime} \nu^{\prime}+\cdots\right) S-\left(\lambda^{\prime} \alpha+\mu^{\prime} \beta\right)^{2}=0 .
$$

This represents a system of circles in which the only restriction is that the center lies on a fixed straight line, the polar of $(\alpha \beta)$.

If $\alpha, \beta, \gamma$ are coincident the circles are concentric with center the pole of $\alpha$.

Passing to the general case in which $\alpha, \beta, \gamma$ form a triangle, we can transform the equation by a linear transformation so that the triangle $\alpha, \beta, \gamma$ is transformed into a self-conjugate triangle of the absolute. Taking this as triangle of reference the equation can be written in the form

$$
P_{1} S-(\lambda x+\mu y+\nu z)^{2}=0,
$$

where

and

$$
S \equiv x^{2}+y^{2}+z^{2}
$$

$$
P_{1} \equiv a \lambda^{2}+b \mu^{2}+c \nu^{2}+2 f \mu \nu+2 g \nu \lambda+2 h \lambda \mu .
$$

The center of the circle is therefore $(\lambda, \mu, \nu)$. When $\lambda: \mu: \nu$ are given, the circle is fixed, hence, with a given point as center there is one and only one circle of the system.

The line equation of the circle (11) is found to be

$$
\left(\lambda^{2}+\mu^{2}+\nu^{2}-P_{1}\right)\left(\xi^{2}+\eta^{2}+\zeta^{2}\right)-(\lambda \xi+\mu \eta+\nu \zeta)^{2}=0,
$$

or

$$
P_{0} \Sigma-(\lambda \xi+\mu \eta+\nu \zeta)^{2}=0
$$


\$14. Locus of limiting points and envelope of limiting lines. The discriminant of (11) is

i. e.,

$$
\left|\begin{array}{rrr}
P_{1}-\lambda^{2} & -\mu \lambda & -\nu \lambda \\
-\lambda \mu & P_{1}-\mu^{2} & -\nu \mu \\
-\lambda \nu & -\mu \nu & P_{1}-\nu^{2}
\end{array}\right|=0
$$

$$
P_{1}^{2}\left(P_{1}-\lambda^{2}-\mu^{2}-\nu^{2}\right)=0 \text { or } \quad P_{1}^{2} P_{0}=0 .
$$

If $P_{1}=0$ the circle (11) degenerates to two coincident lines $\lambda x+\mu y+\nu z=0$. The line equation of the envelope of these limiting lines is therefore

$$
a \xi^{2}+b \eta^{2}+c \zeta^{2}+2 f \eta \zeta+2 g \zeta \xi+2 h \xi \eta=0 .
$$

The point equation of the envelope of limiting lines is therefore

$$
S_{1}{ }^{\prime} \equiv A x^{2}+B y^{2}+C z^{2}+2 F y z+2 G z x+2 H x y=0 .
$$

The locus of the absolute poles of these lines, or centers of the circles which degenerate to coincident lines, is the reciprocal of $S_{1}{ }^{\prime}$ with regard to $S$, and its point equation is

$$
S_{1} \equiv a x^{2}+b y^{2}+c z^{2}+2 f y z+2 g z x+2 h x y=0 .
$$

The circle (12) degenerates to two distinct straight lines, or a point circle, if $P_{0}=0$. The center being $(\lambda, \mu, \nu)$, the equation of the locus of point circles is therefore

$$
S_{0} \equiv S-S_{1}=0 .
$$

The envelope of the axes of the point circles is the reciprocal of (15) with regard to $S$, and its point equation is thus found to be

$$
S_{0}{ }^{\prime} \equiv S_{1}+S_{1}{ }^{\prime}+(1-\Sigma a) S=0 .
$$

$\S 15$. Horocycles. The circle will be a horocycle if the line $\lambda x+\mu y+\nu z=0$ is a tangent to $S$, i. e., if

$$
\lambda^{2}+\mu^{2}+\nu^{2}=0 .
$$

Hence through any point there pass four horocycles of the system. 
$\S 16$. Consider the circles of the system whose centers lie on a given line $l x+m y+n z=0$. We shall call this a row of circles.

We have

$$
l \lambda=m \mu+n \nu=0 .
$$

Eliminating $\nu$, we get

$$
\begin{aligned}
& {\left[n^{2}\left(a \lambda^{2}+2 h \lambda \mu+b \mu^{2}\right)-2 n(g \lambda+f \mu)(l \lambda+m \mu)\right.} \\
& \left.+c(l \lambda+m \mu)^{2}\right] S-[n(\lambda x+\mu y)-(l \lambda+m \mu) z]^{2}=0 .
\end{aligned}
$$

This is a one-parameter system of circles

$$
\begin{aligned}
& {\left[\left(a n^{2}-2 g n l+c l^{2}\right) \lambda^{2}+2\left(h n^{2}-g m n-f n l+c l m\right) \lambda \mu\right.} \\
& \left.\quad+\left(b n^{2}-2 f m n+c m^{2}\right) \mu^{2}\right] S-[(n x-l z) \lambda+(n y-m z) \mu]^{2}=0,
\end{aligned}
$$

having as envelope the conic

$$
\begin{aligned}
& T \equiv\left(A l^{2}+B m^{2}+C n^{2}+2 F m n+2 G n l+2 H l m\right) S \\
& -\Sigma x^{2}\left(c m^{2}-2 f m n+b n^{2}\right)+2 \Sigma y z\left(f l^{2}+a m n-h n l-g l m\right)=0 .
\end{aligned}
$$

This is a two-parameter quadratic system of conics. Two of the foci of $T$ are limiting points of the system of circles and therefore lie on the conic $S_{0}$, and the corresponding directrices touch the conic $S_{0}{ }^{\prime}$; the two corresponding focal lines are limiting lines of the system and therefore touch the conic $S_{1}{ }^{\prime}$, and the corresponding director points lie on the conic $S_{1}$. $\S 17$. The two lines

$\Sigma x^{2}\left(c m^{2}-2 f m n+b n^{2}\right)-2 \Sigma y z\left(f l^{2}+q m n-h n l-g l m\right)=0$, which pass through the point of intersection of $n x-l z=0$ and $n y-m z=0$, i. e., the point $(l, m, n)$, are in fact the tangents from this point to the conic $S_{1}{ }^{\prime}$, and the equation of the conic envelope can be written

$$
\begin{aligned}
\Delta T \equiv\left(A l^{2}+\cdots+2 F m n\right. & +\cdots)\left(\Delta S-S_{1}{ }^{\prime}\right) \\
- & {[x(A l+H m+G n)+\cdots]^{2}=0 . }
\end{aligned}
$$

Hence the conic envelopes $T$ all have double contact with the fixed conic $\Delta S-S_{1}{ }^{\prime}=0$.

If the conic $S^{\prime}$ coincides with the absolute $S$, the conics $T$ are all circles. In this case all the circles of the system have equal radii. 
We shall consider in the next section what rows of circles with equal radii exist in the general system.

$\S 18$. Let $\rho$ be the radius of the circle

Then

$$
P_{1}\left(x^{2}+y^{2}+z^{2}\right)-(\lambda x+\mu y+\nu z)^{2}=0 .
$$

$$
\cos ^{2} \rho=\frac{(\lambda x+\mu y+\nu z)^{2}}{\left(x^{2}+y^{2}+z^{2}\right)\left(\lambda^{2}+\mu^{2}+\nu^{2}\right)}=\frac{P_{1}}{\lambda^{2}+\mu^{2}+\nu^{2}} .
$$

If the center $(\lambda, \mu, \nu)$ is given, the radius is in general determined, so that with a given center there is in general just one circle of the system. $\rho$ will, however, be indeterminate if both

$$
P_{1}=0 \text { and } \lambda^{2}+\mu^{2}+\nu^{2}=0 .
$$

Hence if the center is at one of the four absolute points on the conic $S_{1}$ or $S_{0}$ the radius is indeterminate.

Consider the locus of centers of circles with a given radius. The equation of the locus is

$$
\left(a x^{2}+b y^{2}+c z^{2}+2 f y z+2 g z x+2 h x y\right)-\left(x^{2}+y^{2}+z^{2}\right) \cos ^{2} \rho=0 .
$$

Hence the locus is a conic homothetic with $S_{1}$ and $S_{0}$.

Putting $\cos ^{2} \rho=r$, the values of $r$ for which this conic breaks up into a pair of straight lines are the roots of the equation

$$
\left|\begin{array}{lll}
a-r & h & g \\
h & b-r & f \\
g & f & c-r
\end{array}\right|=0
$$

This equation has three real roots, hence there are three pairs of rows of circles each with equal radii. We shall call the lines of centers of these circles the equiradial axes.

The three pairs of equiradial axes are common chords of $S$ and $S_{1}, i$. e., the pairs of focal lines of the conic $S_{1}\left(\right.$ or $\left.S_{0}\right)$. The axes of these rows of circles are concurrent in three pairs of points which are the director points of $S_{1}$ (or $\left.S_{0}\right)$, or the foci of $S_{1}{ }^{\prime}\left(\right.$ or $\left.S_{0}{ }^{\prime}\right)$.

These results are easily proved also directly by taking as the triangle of reference the common self-conjugate triangle of $S$ and $S_{1}$, so that the equation of the system becomes

$$
\left(a \lambda^{2}+b \mu^{2}+c \nu^{2}\right)\left(x^{2}+y^{2}+z^{2}\right)-(\lambda x+\mu y+\nu z)^{2}=0 .
$$


The three pairs of equiradial axes are then given by

$$
\begin{aligned}
& (a-b) y^{2}-(c-a) z^{2}=0, \\
& (b-c) z^{2}-(a-b) x^{2}=0, \\
& (c-a) x^{2}-(b-c) y^{2}=0,
\end{aligned}
$$

the radii being respectively $\cos ^{-1} \nu a, \cos ^{-1} \nu b, \cos ^{-1} \nu c$.

If $b=c$, the conics $S_{1}$ and $S_{0}$ have double contact with $S$ and are concentric circles. In this case the last two pairs of axes coincide with $x=0$, which is the axis of $S_{1}$ or $S_{0}$, and the first pair reduces to $y^{2}+z^{2}=0$, which consists of the absolute tangents of $S_{1}$ or $S_{0}$.

$\S 19$. The discriminant of the conic envelope $T$ is

where

$$
\Sigma_{1}^{2} \Sigma_{0}=0
$$

$$
\begin{aligned}
& \Sigma_{1} \equiv \Sigma A l^{2}+2 \Sigma F m n, \\
& \Sigma_{0} \equiv\left(\Sigma A l^{2}+2 \Sigma F m n\right)+\left(\Sigma a l^{2}+2 \Sigma f m n\right)-\Sigma l^{2}(\Sigma a-1) .
\end{aligned}
$$

When $\Sigma_{1}=0, T$ degenerates to a point pair. Since $\Sigma_{1}=0$ is the line equation of the conic $S_{1}$, we see that the circles whose centers all lie on a tangent to $S_{1}$ form a coaxial system, the common radical axis being the absolute polar of the point of contact of this tangent.

When $\Sigma_{0}=0, T$ degenerates to a line pair. Since $\Sigma_{0}=0$ is the line equation of the conic $S_{0}$, we see that the circles whose centers all lie on a tangent to $S_{0}$ form a homocentric system, the common homothetic center being the point of contact of this tangent.

$\$ 20$. $T$ will degenerate to a point pair for every row of circles if $\Sigma_{1}$ vanishes identically, $i$. e., if $P_{1}$ is a perfect square. Then all the limiting lines pass through one point, and the locus of point circles $S_{0}$ becomes a circle with this point as center. In this case we can prove that every circle of the system cuts the circle $S_{0}$ orthogonally.

$$
\text { Let } \begin{aligned}
a= & p^{2}, b=q^{2}, c=r^{2}, f=q r, g=r p, h=p q, \text { then } \\
-T & \equiv \Sigma x^{2}(r m-q n)^{2}+2 \Sigma y z(p n-r l)(q l-p m) \\
& \equiv[\Sigma(r m-q n) x]^{2} .
\end{aligned}
$$

Hence the point pair lies on the line $\Sigma(r m-q n) x=0$ which 
passes through the fixed point $(p, q, r)$, the center of the circle $S_{0}$.

The polar of $(p, q, r)$ with regard to the circle

is

$$
(\Sigma p \lambda)^{2} \cdot \Sigma x^{2}-(\Sigma \lambda x)^{2}=0
$$

$$
\Sigma p \lambda \cdot \Sigma p x-\Sigma \lambda x=0 .
$$

Now forming the equation of a pair of common chords of this circle and the circle

$$
S_{0} \equiv(\Sigma p x)^{2}-\Sigma x^{2}
$$

we have

$$
\begin{aligned}
(\Sigma p \lambda)^{2} \cdot \Sigma x^{2}-(\Sigma \lambda x)^{2}+\left[(\Sigma p x)^{2}-\Sigma x^{2}\right](\Sigma p \lambda)^{2} & \\
& =(\Sigma p x)^{2}(\Sigma p \lambda)^{2}-(\Sigma \lambda x)^{2} .
\end{aligned}
$$

Hence one of the common chords is $\Sigma p x \cdot \Sigma p \lambda-\Sigma \lambda x=0$, which is the polar of $(p, q, r)$. Hence the circles cut orthogonally.

$\S 21$. $T$ will degenerate to a line pair for every row of circles of the system if $\Sigma_{0}$ vanishes identically. This is the condition that $P_{0}$ should be a perfect square.

Now every point on a circle is tangentially distant a quadrant from its axis, and if two circles are such that the length of one of their common tangents is a quadrant, say the circles are quadrantal, each of the points of contact of this tangent must lie on the axis of the other circle. The same will be true for the other common tangent belonging to the same pair. If these two tangents intersect in 0 , the polar of 0 with regard to each circle is the axis of the other circle.

Since the line equation of the system of circles is

$$
P_{0} \Sigma-(\lambda \xi+\mu \eta+\nu \zeta)^{2}=0,
$$

the analytical work in deducing the conclusions from the conditions that $P_{0}$ should be a perfect square is exactly the same as that of the last section. Hence if this condition is satisfied, the locus of point circles consists of two coincident straight lines, the envelop of limiting lines is a circle, of which the locus of point circles is the axis, and every circle of the system is quadrantal with respect to this circle.

\footnotetext{
Victoria University College,

Wellington, N. Z., August, 1918.
} 